\title{
Cross-Layer Design for Two-Way Relaying Networks with Multiple Antennas
}

\author{
Lei Wang \\ The Third Research Institute of Ministry of Public Security \\ No.339 Bisheng Road \\ Pudong District, Shanghai, China \\ yoyo604913@163.com
}

\author{
Zhuo Wu \\ Shanghai University \\ No.149 Yanchang Road \\ Zhabei District, Shanghai, China \\ zwu@shu.edu.cn
}

\begin{abstract}
In this paper, we developed a cross-layer design for two-way relaying (TWR) networks with multiple antennas, where two single antenna source nodes exchange information with the aid of one multiple antenna relay node. The proposed cross-layer design considers adaptive modulation (AM) and space-time block coding (STBC) at the physical layer with an automatic repeat request (ARQ) protocol at the data link layer, in order to maximize the spectral efficiency under specific delay and packet error ratio (PER) constraints. An MMSE-interference cancellation (IC) receiver is employed at the relay node, to remove the interference in the fist phase of the TWR transmission. The transmission mode is updated for each phase of the TWR transmission on a frame-byframe basis, to match the time-varying channel conditions and exploit the system performance and throughput gain. Simulation results show that retransmission at the data link layer could alleviate rigorous error-control requirements at the physical layer, and thereby allows higher data transmission. As a result, crosslayer design helps to achieve considerable system spectral efficiency gain for TWR networks, compared to those without cross-layer design.
\end{abstract}

\section{Keywords}

Cross-layer design, two-way relaying, MMSE-interference cancellation, STBC.

\section{INTRODUCTION}

Two-way relaying has attracted much attention recently [1-5], where two source nodes exchange information via a relay node located between them. It has been demonstrated that improved spectral efficiency can be achieved by using physical layer network coding (PNC) to shorten the information exchange process into two phases, the multiple-access (MA) phase and the broadcast (BC) phase [1]. So far, a number of strategies have been devised, including the amplify-and-forward [6] and decode-andforward [1-5] strategies, to form the signals to be transmitted from the relay back to the two source nodes in the BC phase. However, most work on PNC assumes single antenna at the source and the relay nodes [1-5]. Since using multiple-input multiple-output (MIMO) techniques can improve the system capacity and achieve spatial diversity, combining the two-phases two-way relaying and MIMO, such as MIMO-PNC and MIMO-network coding (NC), is worth being investigated.
In [7], a MIMO PNC scheme was proposed to extract the summation and the difference of the information of the two source nodes at the relay with linear MIMO detection scheme. [8] explored a two-step communication protocol with virtual MIMO and network coding. An adaptive network coding scheme for MIMO bidirectional relaying with precoding was proposed in [9] to improve the e2e throughput performance. An optimal transmit strategy for the multiple-input single-output bidirectional broadcast channel was proposed in [10]. However, they all assume symmetric transmission scenarios where the two source nodes use the same modulation schemes, considering only BPSK or QPSK modulation, and the BER at the relay or the e2e BER at the two source nodes is inferior to that of the optimal maximum likelihood (ML) detection.

In practice, besides symmetric transmission, asymmetric transmission where the two source nodes use different modulation schemes is also worth being looked into, since the data rates required by the applications from the two sources or the channel conditions from the two sources to the relay are unlikely to be identical. Some strategies have been proposed for two-way relaying with asymmetric transmission [3, 5]. However, they all considered single-antenna source and relay nodes only.

In addition, link adaptation is critical to obtain reliable communications and maximize throughput of communication systems. Transmitter designs adapted to the varying wireless channel are capable of improving both performance and rate of communication links. In recent years, cross-layer design for wireless communication systems has been paid more and more attention [18-19]. Considering adaptive modulation at the physical layer, along with the scheduling in the data link layer, it has been demonstrated that a cross-layer optimization methodology could improve the system throughput [20]. In [2125], adaptive modulation and coding was considered in a TWR network, showing improved throughput by designing different codebooks under different channel conditions. In [26], cross-layer design was first investigated for a cooperative communication system. However, it was only for a single antenna system, and the cooperative transmission was only for one-way relaying. In [2728], cross-layer design for TWR networks was explored. However, the two-way relaying considered were both three-phase two-way relaying with single antenna nodes, and the analysis was for resource allocation and from an information theoretical point of view.

Therefore, in this paper, we investigate the cross-layer design of a TWR network with two-phase two-way relaying, by considering adaptive modulation and STBC at the physical layer with an ARQ protocol at the data link layer, in order to maximize the spectral efficiency under specific delay and PER constraints. A MMSE-IC detector and joint designing of the constellation mapping for the broadcast signal at the relay are also employed to obtain a more 
efficient relay scheme and further BER performance improvements. The transmission mode is updated for each phase of the TWR transmission on a frame-by-frame basis, to match the time-varying channel conditions and exploit the system performance and throughput gain.

The rest of the paper is organized as follows: In Section II, the system description and the channel mode are described. The proposed cross-layer design is presented in Section III. Simulation results are illustrated in Section IV, and conclusions are drawn in the last section.

\section{SYSTEM MODEL}

\subsection{System Description}

We consider a two-way MIMO relay channel, as shown in Fig.1. Two source nodes, $S_{1}$ and $S_{2}$, each equipped with single antenna, exchange information through the relay node R, equipped with $N_{R}$ antennas. The TWR transmission can be divided into two phases, MA and BC phase, where sources $S_{1}$ and $S_{2}$ transmit their information simultaneously to $\mathrm{R}$ in the MA phase. In the $\mathrm{BC}$ phase where the relaying signal $s_{R}$ is broadcasted to the sources $\mathrm{S}_{1}$ and $\mathrm{S}_{2}$.

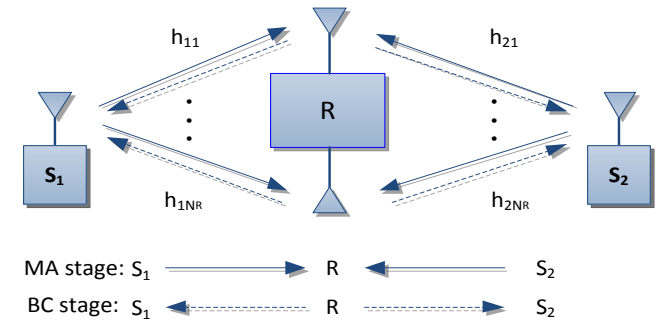

Fig.1 Two-way relaying channel with multiple antennas

The schematic of the proposed cross-layer joint adaptive modulation and ARQ design for TWR is shown in Figure 2. The processing unit at the data link layer is a packet. At the physical layer, in the MA phase, a data packet with $L$ bits from each source node is encoded by cyclic redundancy check (CRC) and sent to the Turbo or convolutional encoder before feeding to the transmit buffer. The modulation selector then selects the modulation mode according to the channel estimates obtained at the receiver. The adaptively modulated signal is then transmitted simultaneously to the relay $\mathrm{R}$ from both source nodes.

Since multiple antennas are equipped at the relay node, the signal processing at the relay node can be divided into two parts: fist, signal detection; second, signal processing. In the signal detection process, the relay node first combines the received signal from both sources on the multiple receive antennas using the maximum ratio combining (MRC), and then detects and demodulates the signal to recover the transmitted signals from $S_{1}$ and $S_{2}$. Since CRC check is employed before the channel encoder, here 4 possible CRC results will be obtained, according to the characteristics of TWR transmission. 1) Both source nodes transmit successfully to the relay, then the relay sends back an acknowledgement (ACK) message to both sources, and both sources will be ready for transmitting the next data packet. 2) If node $S_{1}$ transmits successfully while node $S_{2}$ transmission fails, an ACK is fed back to $S_{1}$ and a no-acknowledgement (NAK) message is sent to node $S_{2}$. Then $S_{1}$ will get ready for the transmitting the next data packet, while a retransmission request is generated by the ARQ generator and sent back to node $S_{2}$ (buffer). The packet is retransmitted until it is correctly received or the maximum number of transmission, $N_{\max }$, is achieved. 3) If node $\mathrm{S}_{2}$ transmits successfully while node $S_{1}$ transmission fails, similar transmission protocol is used as that used in 2). 4) If both nodes' transmission fails, then both nodes will receive a NAK message and begin their retransmission until transmission successes or the maximum number of retransmission is achieved.

When the relay completes signal detection, the signal processing starts to reconstruct a signal and broadcasts back to both source nodes. Conventionally, the XOR processing is used to reconstruct the signal to be transmitted from the relay. While in our paper, a new joint designing of the constellation mapping (JCM) scheme is employed at the relay to achieve further performance gain in the $\mathrm{BC}$ phase. After CRC encoding, the signal is sent to the relay (buffer), adaptively modulated according to the channel states and space-time block coded, before transmitting to both source nodes.

In the $\mathrm{BC}$ phase, both sources receive the data packet from the relay. The received signal is first space-time decoded and then demodulated for CRC checking. If any of the source nodes receive a NAK message, the relay will retransmit the data packet until a successful transmission is achieved at both sources or the maximum number of retransmission is achieved.

\subsection{Channel Model}

As shown in Figure 1, direct link between $S_{1}$ and $S_{2}$ is assumed to be unavailable, and the channel from the source nodes $S_{j}(j=1,2)$ to the relay is assumed to be equal to the channel from the relay to source node $S_{j}(j=1,2)$ due to channel reciprocity [1-8]. All nodes are assumed to operate in the half-duplex mode [6].

In the MA phase, both sources $S_{1}$ and $S_{2}$ transmit their information simultaneously to $\mathrm{R}$. The received signal at the relay can then be written as:

$$
\mathbf{y}_{r}=\mathbf{H s}+\mathbf{n}=\mathbf{h}_{1} s_{1}+\mathbf{h}_{2} s_{2}+\mathbf{n}
$$

where $\mathbf{y}_{r}=\left[\begin{array}{llll}y_{1} & y_{2} & \ldots & y_{N_{R}}\end{array}\right]^{T}$ and $\mathbf{H}=\left[\begin{array}{ll}\mathbf{h}_{1} & \mathbf{h}_{2}\end{array}\right]=\left[\begin{array}{cc}h_{11} & h_{21} \\ \vdots & \vdots \\ h_{1 N_{R}} & h_{2 N_{R}}\end{array}\right]$ represents the channel coefficients between $\mathrm{S}_{1}\left(\mathrm{~S}_{2}\right)$ and $\mathrm{R}$ with $h_{j l} \sim \mathcal{C N}(0,1), j=1,2$ and $l=1, \cdots, N_{R}$, where $\mathcal{C} \mathcal{N}(0, x)$ represents a complex Gaussian distribution with 0 mean and variance $x . \mathbf{s}=\left[s_{1} s_{2}\right]^{T}$ denotes the transmitted symbols from $\mathrm{S}_{1}$ and $\mathrm{S}_{2}$ with $M$-PSK modulation. $\mathbf{n}=\left[n_{1} n_{2} \ldots n_{N_{R}}\right]^{T}$ stands for the i.i.d. additive white Gaussian noise (AWGN) at $\mathrm{R}$ with $n_{l} \sim \mathcal{C} \mathcal{N}\left(0, \sigma^{2}\right), l=1, \cdots, N_{R}$. The relay $\mathrm{R}$ then detects the received signal from $\mathbf{y}_{r}$ to estimate $s_{1}$ and $s_{2}$, and then the detected estimates of $s_{1}$ and $s_{2}$ are mapped into a relaying signal $s_{R}$ in the broadcast (BC) phase. Different from the detection method in [78], an MMSE-Interference Cancellation (IC) detector is proposed and employed here, to remove the multiple access interference $s_{2}$ $\left(s_{1}\right)$ when detecting $s_{1}\left(s_{2}\right)$ at the relay.

In the BC phase where the relaying signal $s_{R}$ is broadcasted to the sources $S_{1}$ and $S_{2}$, compared with the traditional XOR processing in [1], a new JCM for signal $s_{R}$ is also investigated. Details of the JCM design and the MMSE-IC detection can be referred to [29]. Then the relaying message $s_{R}$ is transmitted using STBC [10] to gain spatial diversity, and the received signal at source $S_{j}$ can be expressed as:

$$
y_{j}=\mathbf{h}_{j} s_{R}+\mathbf{n}_{j}, j \in\{1,2\}
$$


where $\mathbf{h}_{j}=\left[\begin{array}{lll}h_{j 1} & \cdots & h_{j k}\end{array}\right]^{T}$ is the same as defined in (1), and $\mathbf{n}_{j}=\left[\begin{array}{llll}n_{j 1} & n_{j 2} \ldots & n_{j N_{R}}\end{array}\right]^{T}$ is the AWGN at source $\mathrm{S}_{j}$ with $n_{j l} \sim \mathcal{C} \mathcal{N}\left(0, \sigma_{j}^{2}\right), l=1, \cdots, N_{R}$. Thanks to the help of its own information $s_{j}$, node $\mathrm{S}_{j}$ can decode the desired bits correctly from $y_{j}$.

For the signal detection at the relay with multiple antennas equipped on it, how to optimally exploit these replicas received from different antennas to enhance the detection performance of PNC is of great interest. From the vector detection perspective, we propose a PNC detection scheme based on MMSE-IC to remove the multiple-access interference (MAI) from $S_{2}\left(S_{1}\right)$ when recovering $S_{1}\left(S_{2}\right)$ at the relay. where $\mathbf{y}^{\prime \prime(0)}=\mathbf{y}_{r}-\mathbf{h}_{2} \tilde{S}_{2}^{(1)}$.

Iteration $k: k=1, \ldots, K$ :

-- Use $\tilde{S}_{1}^{(k)}$ obtained from last iteration to calculate $\mathbf{y}^{\prime(k)}=\mathbf{y}_{r}$ $\mathbf{h}_{1} \tilde{s}_{1}^{(k)}$.

-- Calculate $\tilde{s}_{2}^{(k+1)}$ by

$$
\tilde{s}_{2}^{(k+1)}=\left(\sigma_{2}^{2} \mathbf{I}+\mathbf{h}_{\mathbf{2}}^{H} \mathbf{h}_{\mathbf{2}}\right)^{-\mathbf{1}} \mathbf{h}_{\mathbf{2}}^{H} \mathbf{y}^{\prime(k)}
$$

-- Then $\tilde{s}_{1}^{(k+1)}$ can be obtained by using

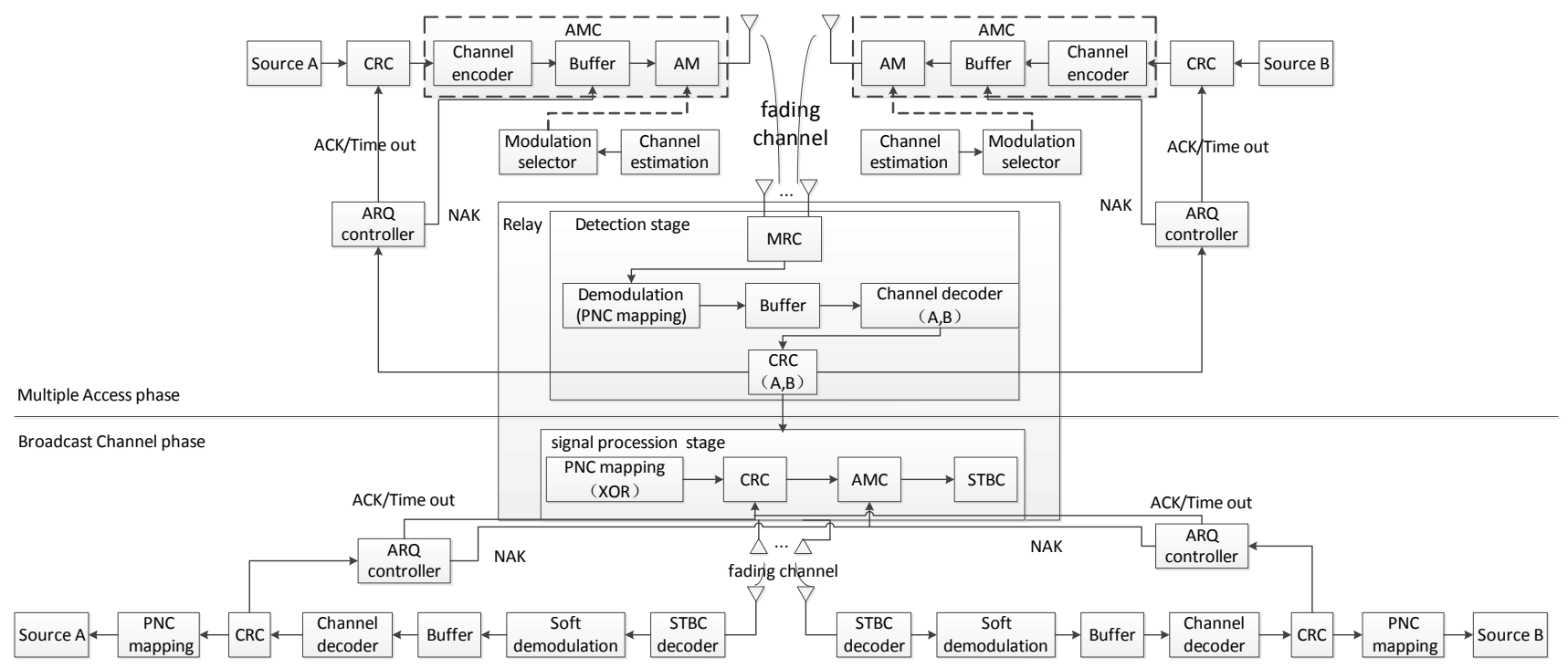

Fig.2 Schematic of the cross-layer design for a TWR network

The MMSE-IC algorithm can be divided into two stages. The MMSE detector is employed in the first stage as:

$$
\tilde{\boldsymbol{s}}^{(\mathbf{0})}=\left[\begin{array}{ll}
\tilde{s}_{1}^{(0)} & \tilde{s}_{2}^{(0)}
\end{array}\right]^{T}=\left(\sigma^{2} \mathbf{I}+\mathbf{H}^{H} \mathbf{H}\right)^{-\mathbf{1}} \mathbf{H}^{H}(\mathbf{H} \mathbf{s}+\mathbf{n})
$$

where estimates of $s_{1}$ and $s_{2}$, denoted as $\widetilde{\mathrm{s}}_{1}^{(0)}$ and $\widetilde{\mathrm{s}}_{2}^{(0)}$, can be obtained, and will be utilized in the initialization of the IC processing carried out in the second stage of the MMSE-IC detection.

\section{Initialization: $k=0$}

-- (ini_i) From the received signal $\mathbf{y}_{r}$, calculate:

$$
\mathbf{y}^{\prime(0)}=\mathbf{y}_{r}-\mathbf{h}_{1} \tilde{s}_{1}^{(0)}
$$

-- (ini_ii) Obtain the estimation of $s_{2}$ in the initialization, $\widetilde{s}_{2}^{(1)}$, using the MMSE processing with $\mathbf{y}^{\prime(0)}$ to obtain $\tilde{s}_{2}^{(1)}$ :

$$
\tilde{s}_{2}^{(1)}=\left(\sigma_{2}^{2} \mathbf{I}+\mathbf{h}_{2}^{H} \mathbf{h}_{2}\right)^{-\mathbf{1}} \mathbf{h}_{2}^{H} \mathbf{y}^{\prime(0)}
$$

-- (ini_iii) The same processing is then performed to obtain $\tilde{s}_{1}^{(1)}$ with

$$
\tilde{s}_{1}^{(1)}=\left(\sigma_{1}^{2} \mathbf{I}+\mathbf{h}_{\mathbf{1}}^{H} \mathbf{h}_{\mathbf{1}}\right)^{-\mathbf{1}} \mathbf{h}_{\mathbf{1}}^{H} \mathbf{y}^{\prime \prime(0)}
$$

$$
\tilde{s}_{1}^{(k+1)}=\left(\sigma_{1}^{2} \mathbf{I}+\mathbf{h}_{\mathbf{1}}^{H} \mathbf{h}_{\mathbf{1}}\right)^{-\mathbf{1}} \mathbf{h}_{\mathbf{1}}^{H} \mathbf{y}^{\prime \prime(k)}
$$

where $\mathbf{y}^{\prime \prime(k)}=\mathbf{y}_{r}-\mathbf{h}_{2} \tilde{S}_{2}^{(k+1)}$

Once the MAI component is removed, the above algorithm will achieve the performance of the optimal ML detection. Meanwhile, a low structural and computational complexity has been retained.

In the $\mathrm{BC}$ phase, how to establish an improved constellation mapping for the network-coded signal broadcasted to the two sources under asymmetric transmission, where the two source nodes use different modulation schemes, is also investigated. For any $M$-PSK constellation with the classical mapping, it is easy to verify that [9]: $p\left(\tilde{s}_{R} \neq s_{R}\right)=\mathcal{Q}\left(\frac{d_{j}\left\|\mathbf{h}_{j}\right\|}{\sigma_{j}} \sin \left(\frac{\pi}{M}\right)\right)$ for $j \in\{1,2\}, k=$ $\left\{1, \cdots, N_{R}\right\}$, where $d_{j}$ denotes the minimum Euclidean distance within the subset of the constellation points based on the side information of the other source node in the two-way relay channel, and $\mathcal{Q}($ ) denotes the Q-function. Given the BER at the relay in the MA phase, denoted by $\lambda$, an end-to-end error occurs if there are no further errors in the BC phase, as two errors will yield a correct decision for a binary system. Therefore, the mapping criterion of the network-coded signal in the BC phase should be designed to minimize the end-to-end BER of the two-way relay channel, as demonstrated in [3]. The mapping design criterion for PNC with multiple antennas can then be represented as: 


$$
\min _{d_{1}, d_{2}} \lambda_{1} \mathcal{Q}\left(\frac{d_{1}\left\|\mathbf{h}_{1}\right\|}{\sigma_{1}} \sin \left(\frac{\pi}{M}\right)\right)+\lambda_{2} \mathcal{Q}\left(\frac{d_{2}\left\|\mathbf{h}_{2}\right\|}{\sigma_{2}} \sin \left(\frac{\pi}{M}\right)\right)
$$

where $\lambda_{i}=1-2 p\left(s_{i} \neq \overline{s_{l}}\right) i=1,2$.

In the following figure, Fig.3, we plot the end-to-end average BER at the source nodes in asymmetric transmission scenarios, using the proposed MMSE-PIC detector with $K=1$ and the joint constellation mapping scheme for the TWR signal in the BC phase. 4 antennas are assumed to be equipped at the relay, and flat Rayleigh fading channel is assumed as the fading channel model. Simulation results demonstrate that further performance improvement can be achieved by using the joint constellation mapping scheme. Details of the JCM scheme can be referred to [29].

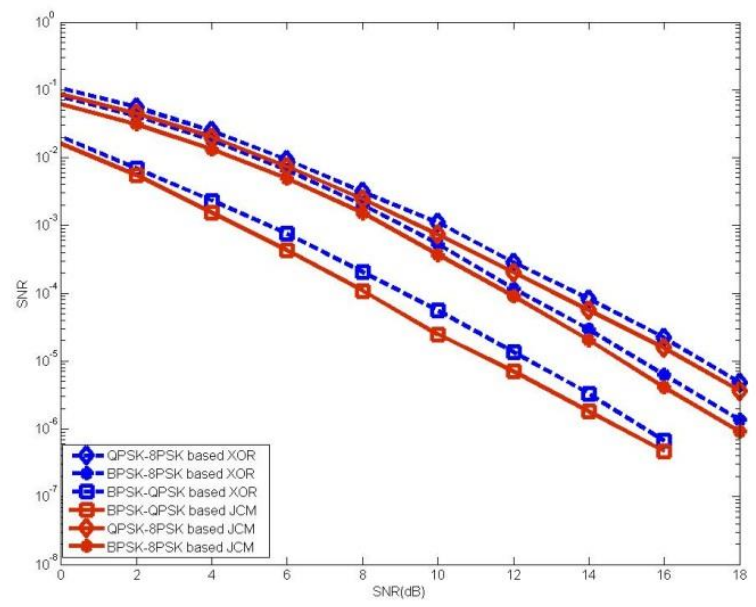

Fig.3 End-to-end performance comparison of asymmetric transmission between JCM and DF-XOR [2] schemes

\section{CROSS-LAYER DESIGN FOR TWR}

A cross-layer design methodology to include adaptive modulation at the physical layer and ARQ at the data link layer is investigated for a TWR network with multiple antennas.

At the physical layer, the transmission is carried out on a frameby-frame basis. Each frame contains a fixed number of symbols. Using adaptive modulation, different transmission mode results in different transmission rates and spectral efficiency. As mentioned above, both symmetric and asymmetric transmission modes for both source nodes to use the same or different modulation schemes are considered in this paper. Therefore, the following six transmission modes are considered in the cross-layer design, as shown in Table 1.

\section{Table 1. Transmission modes description}

\begin{tabular}{|l|l|l|l|l|l|l|}
\hline $\begin{array}{l}\text { TM } \\
\text { ode }\end{array}$ & mode1 & mode2 & mode3 & mode4 & mode5 & mode6 \\
\hline $\begin{array}{l}\text { Mod } \\
\text { ulati } \\
\text { on }\end{array}$ & BPSK & BPSK & QPSK & BPSK & QPSK & 8PSK \\
\hline $\begin{array}{l}\text { Data } \\
\text { rate }\end{array}$ & $\begin{array}{l}1 \mathrm{bit} \\
/ \mathrm{sym}\end{array}$ & $\begin{array}{l}1.5 \mathrm{bit} \\
/ \mathrm{sym}\end{array}$ & $\begin{array}{l}2 \mathrm{bit} \\
/ \mathrm{sym}\end{array}$ & $\begin{array}{l}2 \mathrm{bit} \\
/ \mathrm{sym}\end{array}$ & $\begin{array}{l}2.5 \mathrm{bit} \\
/ \mathrm{sym}\end{array}$ & $\begin{array}{l}3 \mathrm{bit} \\
/ \mathrm{sym}\end{array}$ \\
\hline
\end{tabular}

The aim of cross-layer design is to design an optimum adaptive modulation scheme at the physical layer with the aid of ARQ at the data link layer to dynamically adjust the modulation level, so as to maximize the system throughput and spectral efficiency, under the constraints of delay caused by retransmission and the overall system performance defined by $P_{\text {loss }}$, which is the worst packet loss probability that the system could tolerate required by quality of service of the interested application after $N_{\max }$ retransmissions.

Suppose that the instantaneous PER is guaranteed to be no greater $P_{0}$ than for any transmission mode, then after $N_{\max }$ retransmissions at the data link layer, the PER should be no great than $P_{0}^{N_{\max }+1}$. It is easy to derive that

$$
P_{0}^{N_{\text {max }}+1} \leq P_{\text {loss }}
$$

This gives that

$$
P_{0} \leq P_{\text {loss }}^{\frac{1}{N_{\max }+1}}
$$

Therefore, we can define the target PER, i.e. the upper bound of the instantaneous PER from (10) as

$$
P_{0} \leq P_{\text {target }}:=P_{\text {loss }}^{\frac{1}{N_{\max }+1}}
$$

It is not difficult to observe that $P_{\text {target }}$ is determined by the system requirements on error performance and delay.

For the six transmission modes listed in table 1, the end-to-end PER of each mode is obtained by using the MMSE-IC detection at the relay node and the JCM scheme described in the previous section. 4 antennas are assumed to be equipped at the relay, and flat Rayleigh fading channel is assumed as the fading channel model.

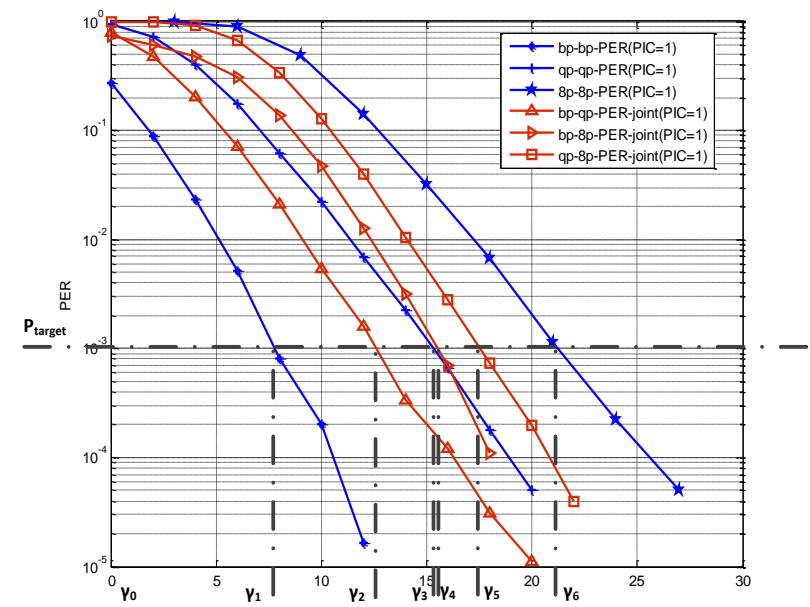

Fig.4 End-to-end PER and SNR regions for all transmission modes

For the TWR transmission, the instantaneous PER of a chosen transmission mode can be determined by the received SNR $\gamma$. Therefore, we can use the received SNR to select the transmission modes. Hence, in Fig.4, it not only shows the PER of all the six transmission modes, but also provides the region boundaries of the received SNR $\left\{\gamma_{0}, \gamma_{1}, \gamma_{2}, \gamma_{3}, \gamma_{4}, \gamma_{5}, \gamma_{6}\right\}$ for different transmission modes, according to the required PER $\leq P_{\text {target }}$ 
(here we set $P_{\text {target }}=0.001$ ). That is, each SNR region corresponds to one transmission mode, e.g. if $\gamma \in\left[\gamma_{m}, \gamma_{m+1}\right)$, the signals should be transmitted in mode $m$. Note that, no transmission will be chosen when $\gamma_{0} \leq \gamma<\gamma_{1}$.

In the Additive White Gaussian Noise (AWGN) channel, for the uncoded system, the closed-form PER expression could be referred to [55, Lei's thesis], but in a rather cumbersome form. However, in the fading channel case, there is not such expression to accurately describe the relationship between $\gamma$ and PER. To simplify the analysis of the adaptive modulation TWR system here, we use the approximate PER expression, as already used in [56-58, Lei's thesis]. Therefore, the PER for each transmission mode $m$ is represented as

$$
\operatorname{PER}_{n}(\gamma) \approx\left\{\begin{array}{cc}
1, & \text { if } 0<\gamma<\gamma_{p n} \\
a_{n} \exp \left(-g_{n} \gamma\right) & \text { if } \gamma \geq \gamma_{p n}
\end{array}\right.
$$

where $a_{n}, g_{n}$, and $\gamma_{p n}$ are dependent on the transmission mode, which can be pre-calculated by using the MMSE-IC estimation results, as shown in Fig.4 and the approximate PER in (13), as shown in Table 2:

Table 2. PER's fitting parameters of six transmission modes

\begin{tabular}{|c|l|l|l|l|l|l|}
\hline $\begin{array}{l}\text { Tx } \\
\text { mode }\end{array}$ & mode1 & $\begin{array}{l}\text { mode } \\
2\end{array}$ & $\begin{array}{l}\text { mode } \\
3\end{array}$ & $\begin{array}{l}\text { mode } \\
4\end{array}$ & $\begin{array}{l}\text { mode } \\
5\end{array}$ & $\begin{array}{l}\text { mode } \\
6\end{array}$ \\
\hline $\begin{array}{l}\text { Modu } \\
\text { lation }\end{array}$ & $\begin{array}{l}\text { BPSK } \\
\text { BPSK }\end{array}$ & $\begin{array}{l}\text { BPSK } \\
-\end{array}$ & $\begin{array}{l}\text { QPSK } \\
-\end{array}$ & $\begin{array}{l}\text { BPSK } \\
\text { QPSSK }\end{array}$ & $\begin{array}{l}\text { QPSK } \\
\text {-8PSK }\end{array}$ & $\begin{array}{l}\text { 8PSK- } \\
8 P S K\end{array}$ \\
\hline$a_{n}$ & 0.426 & 1.593 & 2.076 & 2.756 & 4.543 & 5.025 \\
\hline$g_{n}$ & 0.796 & 0.586 & 0.495 & 0.494 & 0.452 & 0.383 \\
\hline
\end{tabular}

With the analysis on the fitting parameters of $a_{n}, g_{n}$, and $\gamma_{p n}$ and $P_{\text {target }}\left(N_{\max }\right)$, it is shown that $P_{\text {target }}\left(N_{\max }\right)$ is a quite important parameter in the cross-layer design. It delivers the worst packet loss probability $P_{\text {loss }}$ and maximum delay $N_{\max }$ which the data link layer could tolerate to the physical layer, so as to control the selection of the transmission modes. Note that the boundary points for each transmission mode are the minimum SNRs required to achieve PER $\leq P_{\text {target }}$ for transmission mode $m$. Given the $P_{\text {target }}$ in (11), the boundary points could be worked out from (12). After iteration, we could obtain

$$
\begin{gathered}
\gamma_{0}=0 \\
\gamma_{n}=\frac{1}{g_{n}} \ln \left(\frac{a_{n}}{P_{\text {target }}}\right), n=1,2, \ldots, M \\
\gamma_{n+1}=+\infty
\end{gathered}
$$

Replace $a_{n}, g_{n}$ and $P_{\text {target }}\left(N_{\max }\right)$ into (14), we could obtain the boundary points for all transmission modes in the case of $P_{\text {loss }}$ and $N_{\max }$. The following table gives the boundary points of SNR for different transmission modes with different numbers of retransmission.

\section{SIMULATION RESULTS}

The proposed cross-layer design for TWR networks with multiple antennas is evaluated, of which the simulation results are shown in the following. We use an independent flat Rayleigh fading
Table 3. Switch boundaries under different $N_{\max }$

\begin{tabular}{|c|c|c|c|c|c|c|}
\hline$N_{\max }$ & $\begin{array}{c}\gamma_{1}(\mathrm{~dB}) \\
\text { BPSK- } \\
\text { BPSK }\end{array}$ & $\begin{array}{c}\gamma_{2}(\mathrm{~dB}) \\
\text { BPSK- } \\
\text { QPSK }\end{array}$ & $\begin{array}{l}\gamma_{3}(\mathrm{~dB}) \\
\text { QPSK- } \\
\text { QPSK }\end{array}$ & $\begin{array}{l}\gamma_{4}(\mathrm{~dB}) \\
\text { BPSK- } \\
8 \text { PSK }\end{array}$ & $\begin{array}{l}\gamma_{5}(\mathrm{~dB}) \\
\text { QPSK- } \\
\text { 8PSK }\end{array}$ & $\begin{array}{c}\gamma_{6}(\mathrm{~dB}) \\
8 P S K- \\
8 P S K\end{array}$ \\
\hline 0 & 7.6 & 12.6 & 15.4 & 16.0 & 18.6 & 22.2 \\
1 & 7.0 & 11.4 & 14.0 & 14.8 & 16.3 & 19.8 \\
2 & 6.8 & 10.8 & 13.7 & 14.3 & 15.9 & 19.6 \\
3 & 6.3 & 10.7 & 13.5 & 14.1 & 14.9 & 19.0 \\
\hline
\end{tabular}

channel model. The channel has identical distribution, and remains invariant during one frame, but varies from one frame to another as in slow time-varying channels. Assume synchronization and channel estimation are perfect at each receiver, and the corresponding mode selection can be fed back to each transmitter without error and delay.

The system requires the target PER of $P_{\text {target }}=0.001$, and each packet includes 200 information bits. For simplification, we use stop-and-wait ARQ [30] at the data link layer. The numbers of retransmission considered are $N_{\max }=0,1,2,3$.

In Fig.5, the PER performance of all the six transmission modes considered in this paper is presented with retransmission of $N_{\max }=1$. Compared to the PER performance shown in Fig. 3 with no retransmission, it could be observed that the PER of all transmission modes is significantly decreased. The performance improvement provided by one time retransmission is different in different transmission modes, for both symmetric and asymmetric transmission of the source nodes.

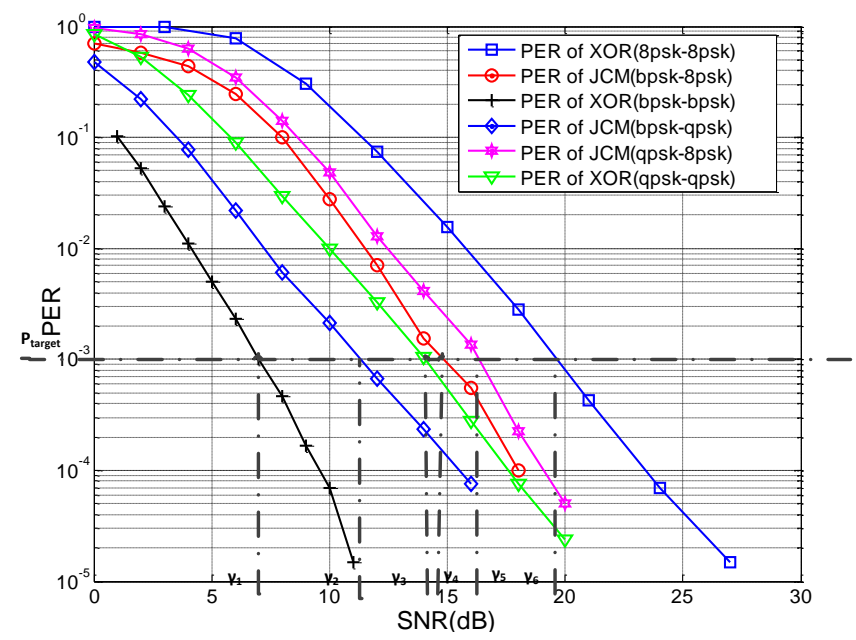

Fig.5 PER performance and SNR regions for six transmission modes with $\mathrm{N}_{\text {max }}=1$

The spectral efficiency of a TWR network with multiple antennas with our proposed cross-layer design is shown in Fig.6, with different numbers of retransmission of $N_{\max }=0,1,2,3$. It is shown that the system spectral efficiency increases with the increasing of retransmission times. However, the improvement of spectral efficiency decreases as the number of retransmission increases. As $N_{\max }$ increases, the target PER at the physical layer, 
$P_{\text {target }}:=P_{\text {loss }}^{\frac{1}{N_{\max }+1}}$ increases as well, which will relax the stringent PER requirement at the physical layer, so as to increase the value of the switching boundaries of SNR for different transmission modes and improve the spectral efficiency of the system. However, increasing $N_{\max }$ means longer delay and buffer size, therefore, the number of retransmission should be carefully chosen according to the QoS and delay requirement.

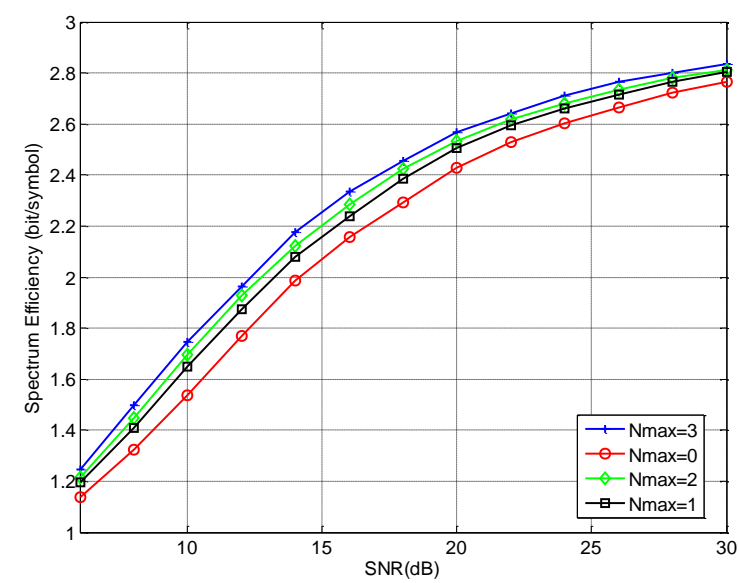

Fig.6 Spectral efficiencies of cross-layer design for physical layer network coding system with $N_{\text {max }}$ varying from 0 to 3

Fig. 7 compares the spectral efficiency of the cross-layer design with adaptive modulation $\left(N_{\max }=1\right)$ and that of adaptive modulation without cross-layer design $\left(N_{\max }=0\right)$. We find that over the entire SNR region, the spectral efficiency increases with increasing SNR, and tends to the maximum spectral efficiency of all the available transmission modes, as shown in Table 2, i.e. 3 bits/symbol. The improvement of using cross-layer design is obvious over that of no cross-layer design. The spectral efficiency improvement is more significant in the low SNR region, since in low SNR region, the PER is comparatively higher, and the retransmission could correct more errors than in the high SNR region.

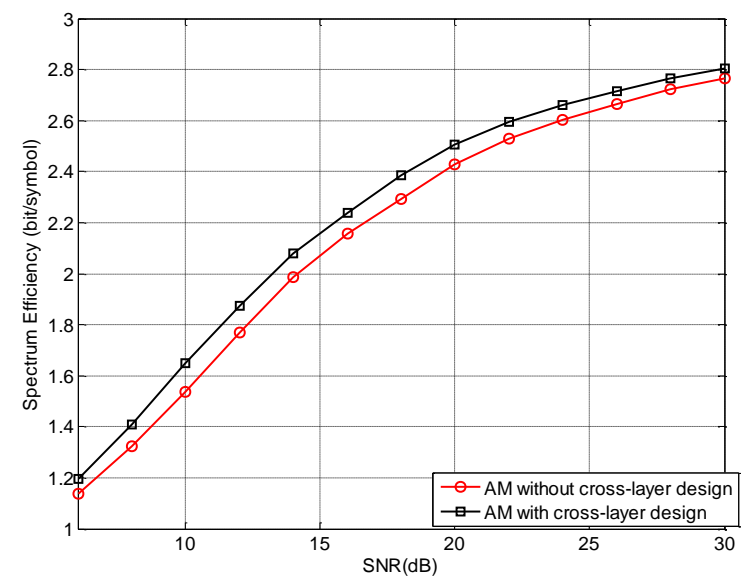

Fig.7 Spectral efficiencies for physical layer network coding system with and without cross-layer design

\section{CONCLUSIONS}

In this paper, we developed a cross-layer design for two-way relaying (TWR) networks with multiple antennas. The proposed cross-layer design employed adaptive modulation and space-time block coding at the physical layer with an automatic repeat request protocol at the data link layer, in order to maximize the spectral efficiency under specific delay and PER constraints. The proposed MMSE- IC receiver at the relay node could remove the interference in the fist phase of the TWR transmission, and therefore improve the PER performance for all transmission modes at the physical layer. The transmission mode is updated for each phase of the TWR transmission on a frame-by-frame basis, to match the time-varying channel conditions and exploit the system performance and throughput gain. Simulation results showed that cross-layer design could help to achieve considerable system spectral efficiency gain for TWR networks, compared to those without cross-layer design.

\section{ACKNOWLEDGMENTS}

This work was supported by Shanghai leading academic discipline project under Grant Nos.S30108, 08DZ2231100, Shanghai Natural Science Foundation under Grant No. 14ZR1415100, key laboratory of specialty fiber optics and optical access networks, Shanghai University, under Grant SKLSFO2012-04. This work was also supported by Research a Security Mechanism for Passive UHF RFID Tag Chip Based On Communication Channel, the National Natural Science Fund under Grant No. 61204025.

\section{REFERENCES}

[1] S. Zhang, S. C. Liew, and P. P. Lam, "Physical layer network coding," in Proc. MobiCom'06, pp 358-365, New York, NY, USA, 2006.

[2] P. Popovski, H. Yomo. "Physical network coding in twoway wireless relay channels", in Proc. IEEE ICC 2007, pp. 707-712, 2007.

[3] Z. Y. Chen, H. L, W. B. Wang. "A novel decoding-andforward scheme with joint modulation for two-way relay channel", IEEE Communications Letters, vol. 14, no. 12, pp. 1149-1151, 2010.

[4] T. Koike-Akino, P. Popovski and V. Tarokh, "Optimized constellations for two-way wireless relaying with physical network coding," IEEE Journal on Selected Areas in Comm., vol. 27, pp. 773-787, 2009.

[5] C. Pan, J. Zheng, "Mapping codebook-based physical network coding for asymmetric two-way Relay Channels", in Proc. IEEE ICC 2010, pp. 1-5, 2010.

[6] J. Joung and Ali H. Sayed, "Multiuser two-way amplify-andforward relay processing and power control methods for beamforming systems", IEEE Trans. Signal Processing, vol.58, no.3, pp.1833-1846, Mar. 2010.

[7] S. Zhang and S. C. Liew, "Physical layer network coding with multiple antennas," in Proc. IEEE WCNC, Apr. 2010.

[8] D. Xu, Z. Bai, A. Waadt, G. H. Bruck, and P. Jung, "Combining mimo with network coding: A viable means to provide multiplexing and diversity in wireless relay networks," in Proc. IEEE ICC, May 2010. 
[9] T. Koike-Akino, "Adaptive Network Coding in Two-Way Relaying MIMO Systems", in Proc. IEEE GLOBECOM 2010, pp. 1-6, 2010.

[10] T.J.Oechtering, R. F. Wyrembelski, and H. Boche, "Multiantenna Bidirectional Broadcast Channels-Optimal Transmit Strategies", IEEE Trans. Signal Processing, vol.57, no.5, pp.1948-1958, May 2009.

[11] H. Degenhardt, T.Unger, A.Klein, "Self-interference MMSE filter design for a cellular multi-antenna two-way relaying scenario", in Proc. The Eighth International Symoposium on Wireless Communication Systems ( ISWCS '11), Nov. 2011.

[12] A. Goldsmith, Wireless Communications. New York, NY: Cambridge, 2005.

[13] H. Jafarkhani, Space-time coding: theory and practice. Cambridge, U.K.: Cambridge Univ. Press, 2005.

[14] D. Yoon, K. Cho, "On the general BER expression of oneand two- dimensional ampltude modulations," IEEE Trans. Commun. 50 (2002) 1074-1080.

[15] Qingwen Liu, Shengli Zhou, "Cross-layer combining of adaptive modulation and coding with truncated ARQ over wireless links," IEEE Trans. Wireless Commun. 3 (5) (2004) 1746-1755.

[16] A.J. Goldsmith, S.-G. Chua, "Adaptive coded modulation for fading channels,” IEEE Trans. Commun. 46 (1998) 595-602.

[17] K.J. Hole, H. Holm, G.E. Oien, “Adaptive multidimensional coded modulation over flat fading channels," IEEE J. Select. Areas Commun. 18 (2000) 1153-1158.

[18] Srivastava V, Motani M. Cross-layer design: A Transactions on Communications, 2005, 43(12): survey and the road ahead. IEEE 112-119.

[19] L. Zhang and W. Heng, "Cross-layer adaptive modulation and coding design for space-time block coded MIMOOFDM systems", Computer Communications, vol.32, pp.540-545, 2009.
[20] W. Huang, K.B. Lataief, “A cross-layer resource allocation and scheduling for multiuser space-time block coded MIMOOFDM systems", in ICC 2005.

[21] T. Koike-Akino, P. Popovski, and V. Tarokh, "Denoising maps and constellations for wireless network coding in twoway relaying systems,'IEEE GLOBECOM, New Orleans, U.S.A., Nov.-Dec. 2008.

[22] T. Koike-Akino, P. Popovski and V. Tarokh, "Optimized constellations for two-way wireless relaying with physical network coding," IEEE JSAC, vol. 27, no. 5, pp. 773-787, June 2009.

[23] T. Koike-Akino, P. Popovski, and V. Tarokh, "Denoising strategy for convolutionally-coded bidirectional relaying," IEEE ICC, Dresden, Germany, June 2009.

[24] T. Koike-Akino, P. Popovski, and V. Tarokh, "Adaptive modulation and network coding with optimized precoding in two-way relaying," IEEE GLOBECOM, Honolulu, Hawaii, Dec. 2009.

[25] T. Koike-Akino, "Adaptive Network Coding in Two-Way Relaying MIMO Systems," GLOBECOM 2010, 2010 IEEE Global Telecommunications Conference, 6-10 Dec. 2010.

[26] Jalil Seifali Harsini, F Lahouti, M Levorato and M Zorzi. "Analysis of Non-Cooperative and Cooperative Type II Hybrid ARQ Protocols with AMC over Correlated Fading Channels" IEEE TRANSACTIONS ON WIRELESS COMMUNICATIONS, VOL. 10, NO. 3, MARCH 2011.

[27] C. Lin, Y. Liu, and M. Tao, "Cross-Layer Resource Allocation of Two-Way Relaying for Statistical Delay-QoS Guarantees", in Proc. IEEE ICC, Ottawa, Canada, Jun. 2012.

[28] C. Lin, et al, "Cross-Layer Optimization of Two-Way Relaying for Statistical QoS Guarantees", accepted in IEEE Journal on Selected Areas in Comm, 2012. Digital Object Identifier: 10.1109/JSAC.2013.130820.

[29] L. Wang, Z. Wu, "Signal Detection and Joint Constellation Modulation for Two-Way Relaying with Multiple Antennas", in IEEE WCNC 2013.

[30] A. S. Tanenbaum, Computer Networks, Prentice-Hall, 1988. 